\title{
HELMHOLTZ EQUATION IN UNBOUNDED DOMAINS: SOME CONVERGENCE RESULTS FOR A CONSTRAINED OPTIMIZATION PROBLEM
}

\author{
GIULIO CIRAOLO
}

\begin{abstract}
We consider a constrained optimization problem arising from the study of the Helmholtz equation in unbounded domains. The optimization problem provides an approximation of the solution in a bounded computational domain. In this paper we prove some estimates on the rate of convergence to the exact solution.
\end{abstract}

\section{INTRODUCTION}

In this paper, we consider a constrained optimization problem which arises from the computational study of wave propagation in unbounded domains. We are interested in a classical scattering problem, which can be stated as follows. Let $D \subset \mathbb{R}^{d}, d \geq 2$, be a bounded domain and let $u$ be the solution of

$$
\begin{cases}\Delta u+k^{2} u=0, & \text { in } \mathbb{R}^{d} \backslash \bar{D}, \\ u=f, & \text { on } \partial D, \\ \lim _{r \rightarrow+\infty} r^{\frac{d-1}{2}}\left(\frac{\partial u}{\partial r}-i k u\right)=0 . & \end{cases}
$$

It is well-known that the solution of problem (1.1) can be written explicitly in terms of layer potentials (see $\mathrm{AK}$ for instance). A challenging problem in real applications is how to approximate the solution of (1.1) in a bounded computational domain $\Omega$, with $D \subset \Omega$. Usually, the goal is to prescribe transparent boundary conditions on $\partial \Omega$ in such a way that the corresponding solution approximates the exact solution on a good fashion.

Many methods have been studied and the research on this topic is still very active (see for instance [Be, Ber, CGS, EM, Gi, GrK, Har, Har, KG, MTH, [SS] and references therein).

In a recent paper [CGS], the authors studied a new approach to the problem of transparent boundary conditions which is based on the minimization of an integral functional arising from the radiation condition at infinity. The

1991 Mathematics Subject Classification. 35J05, 35P25, 65N15.

Key words and phrases. Helmholtz equation, Transparent boundary conditions, Minimization of integral functionals.

The paper was completed while the author was visiting "The Institute for Computational Engineering and Sciences" (ICES) of The University of Texas at Austin, and he wishes to thank the Institute for hospitality and support. The author has been also supported by the NSF-DMS Grant 1361122, the "Gruppo Nazionale per l'Analisi Matematica, la Probabilità e le loro Applicazioni" (GNAMPA) of the Istituto Nazionale di Alta Matematica (INdAM) and the Firb project 2013 "Geometrical and Qualitative aspects of PDE". 
approach in CGS works under quite general assumptions on the index of refraction. Indeed, it applies to the study of the Helmholtz equation

$$
\Delta u+k^{2} n(x)^{2} u=0 \quad \text { in } \mathbb{R}^{d} \backslash \bar{D},
$$

where the index of refraction $n$ may have some angular dependency at infinity, i.e. $n(x) \rightarrow n_{\infty}(x /|x|)$ as $|x| \rightarrow+\infty$, as well as some (unbounded) perturbations. The novelty of the method is that it is not based on the knowledge of the exact solution in some exterior domain, but it relies on a different formulations of the radiation condition at infinity (see [PV]); indeed, under suitable assumptions on $n$, the uniqueness of the solution for (1.2) is guaranteed by the radiation condition

$$
\int_{\mathbb{R}^{d} \backslash D}\left|\nabla u-i k n u \frac{x}{|x|}\right|^{2} \frac{d x}{1+|x|}<\infty .
$$

When a computational domain $\Omega$ is considered, one can try to approximate the solution of (1.2)-(1.3) by the minimizer $u_{\Omega}$ of the following constrained optimization problem

$$
\begin{aligned}
\min J_{\Omega}(v)= & \int_{\Omega \backslash D}\left|\nabla v-i k n v \frac{x}{|x|}\right|^{2} \frac{d x}{1+|x|}, \\
& \text { where } \Delta v+k^{2} n(x)^{2} v=0 \quad \text { in } \Omega \backslash \bar{D}, v=f \text { on } \partial D .
\end{aligned}
$$

In CGS] it was proven that, if $\Omega=B_{R}$ (a ball of radius $R$ centered at the origin and containing the scatterer), then the minimizer $u_{B_{R}}$ of (1.4) converges in $H_{l o c}^{1}$ norm to the solution of (1.2)-(1.3) as $R \rightarrow+\infty$.

As already mentioned, this approach works under very general assumptions on $n$ which are not covered by classical methods available in literature (at list in a standard way). Other advantages of this method are: (i) it works for very general choices of $n$ and $\Omega$ [CGS]; (ii) it is of easy implementation since it consists in minimizing a quadratic functional subject to a linear constrain; (iii) it is suitable to be generalized to more general settings, such as the waveguide's case by using the results in [Ci1]- CM2], [MS].

If one considers the problem in its full generality, a rigorous study of the rate of convergence properties of this problem appears to be difficult. In the present paper, we shall study the rate of convergence of this approach in the simplest case possible: $n \equiv 1, d=2, D=B_{R_{0}}$ and $\Omega=B_{R}$, with $R_{0}<R$. It results that the rate of the $H_{l o c}^{1}$ norm convergence to the exact solution is $R^{-1}$ as $R \rightarrow+\infty$. Compared to the existing methods in literature, for $n \equiv 1$ this approach gives a slower rate of convergence. However, we believe that the understanding of this simple case gives a hint on the rate of convergence for much more general indexes of refraction, for which the method is more suitable (see also the numerical studies in [CGS2]).

The paper is organized as follows. In Section 2 we state the problem, recall the main results in [CGS] and prove some preliminary results. In Section 3, we find an explicit representation of the solution by means of Fourier series. As a consequence, we obtain the convergence estimates. 


\section{Preliminaries}

In this section we introduce some notation and recall some results from [CGS] which will be useful in the rest of the paper. Some preliminary results will also be proven.

Let $R_{0}>0$ be fixed and let $\psi$ be the solution of

$$
\begin{cases}\Delta \psi+k^{2} \psi=0 & \text { in } \mathbb{R}^{2} \backslash \bar{B}_{R_{0}} \\ \psi=f & \text { on } \partial B_{R_{0}} \\ \lim _{r \rightarrow+\infty} r^{\frac{1}{2}}\left(\frac{\partial \psi}{\partial r}-i k \psi\right)=0, & \end{cases}
$$

where $r:=|x|, f \in L^{2}\left(\partial B_{R_{0}}\right)$. We consider the polar coordinates $x=$ $r(\cos \omega, \sin \omega)$, where $r=|x|$ and $\omega \in[0,2 \pi)$, so that

$$
\Delta u=\frac{1}{r} \frac{\partial}{\partial r}\left(r \frac{\partial u}{\partial r}\right)+\frac{1}{r^{2}} \frac{\partial^{2} u}{\partial \omega^{2}}
$$

and

$$
\nabla u=\frac{\partial u}{\partial r} \mathbf{e}_{r}+\frac{1}{r} \frac{\partial u}{\partial \omega} \mathbf{e}_{\omega}
$$

where $\mathbf{e}_{r}=x /|x|$ and $\mathbf{e}_{\omega}=\left(-x_{2}, x_{1}\right) /|x|$.

By separating the variables, a solution $u$ of $\Delta u+k^{2} u=0$ can be written as

$$
u(r, \omega)=\sum_{z \in \mathbb{Z}}\left[a_{n} J_{n}(k r)+b_{n} Y_{n}(k r)\right] e^{i n \omega} ;
$$

here $J_{n}(r)$ and $Y_{n}(r)$ are, respectively, the Bessel and Neumann functions of order $n$ and they satisfy (see [AS])

$$
\frac{1}{r}\left(r \phi^{\prime}(r)\right)^{\prime}+\left(1-\frac{n^{2}}{r^{2}}\right) \phi(r)=0, \quad r \in(0,+\infty) .
$$

We also recall that the Hankel functions of order $n$ and type 1 and 2 are given by

$$
H_{n}^{(1)}(r)=J_{n}(r)+i Y_{n}(r), \quad H_{n}^{(2)}(r)=J_{n}(r)-i Y_{n}(r) .
$$

By using these notations, the solution $\psi$ to problem (2.1) is given by

$$
\psi(x)=\sum_{n \in \mathbb{N}} f_{n} \frac{H_{n}^{(1)}(k|x|)}{H_{n}^{(1)}\left(k R_{0}\right)} e^{i n \omega},
$$

where

$$
f_{n}=\frac{1}{2 \pi} \int_{0}^{2 \pi} f(\omega) e^{-i n \omega} d \omega .
$$

Here, we used the fact that $H_{n}^{(1)}$ satisfies

$$
\lim _{r \rightarrow \infty} r^{\frac{1}{2}}\left(H_{n}^{(1) \prime}(r)-i H_{n}^{(1)}(r)\right)=0,
$$

which implies that the outgoing solution of (2.1) can be written in terms of $H_{n}^{(1)}, n \in \mathbb{N}$.

In CGS the authors proposed a method for approximating $\psi$ on

$$
\mathcal{A}_{R}:=B_{R} \backslash \bar{B}_{R_{0}},
$$


which is based on the following minimization problem:

$$
\begin{aligned}
\text { Minimize } J_{R}(u):= & \int_{\mathcal{A}_{R}}\left|\nabla u-i k u \frac{x}{|x|}\right|^{2} d x, \\
& \quad \text { where } \Delta u+k^{2} u=0 \text { in } \mathcal{A}_{R}, u=f \text { on } \partial B_{R_{0}} .
\end{aligned}
$$

We will denote by $u_{\mathcal{A}_{R}}$ the minimizer of (2.8) (see [CGS] for the existence and uniqueness of the minimizer). As already mentioned in the introduction, the problem considered in [CGS] is much more general than problem (2.1) both for the choice of the domain and for the coefficient $n$, which here is fixed to be $n \equiv 1$ while in [CGS] may have angular dependence as well as perturbations.

The reader will notice that the functional in (2.8) differs from the one mentioned in the Introduction in the absence of the weight $(1+|x|)^{-1}$. However, the two integral formulations of the radiation condition are equivalent when $n \equiv 1$, as follows from an asymptotic expansion at infinity of the solution (see also Section 3 in [CGS]). The choice of the functional without the weight is just to simplify the computations. In the present paper we will deal only with a constant index of refraction, since in this case we know the explicit solution and accurate convergence results can be obtained analytically.

The main results in CGS were: (i) the existence and uniqueness of the minimizer $u_{\mathcal{A}_{R}}$ for (2.8); (ii) $u_{\mathcal{A}_{R}} \rightarrow \psi$ in $H_{l o c}^{1}$ norm as $R \rightarrow+\infty$. We summarize these results in the following theorem (the results are stated for the particular case studied in this paper).

Theorem 2.1. Let $\psi$ be given by (2.6). We have the following results

(i) for any $R>R_{0}$ there exists a unique minimizer $u_{\mathcal{A}_{R}}$ of Problem (2.8);

(ii) $u_{\mathcal{A}_{R}}$ is a solution of

$$
\Delta u+k^{2} u=0 \quad \text { in } \mathcal{A}_{R}, \quad u=f \text { on } \partial B_{R_{0}} ;
$$

(iii) the minimizer of (2.8) converges to $\psi$ as $R \rightarrow+\infty$ in the $H_{l o c}^{1}$ norm, that is: for any fixed $\rho>R_{0}$, we have that

$$
\lim _{R \rightarrow+\infty}\left\|u_{\mathcal{A}_{R}}-\psi\right\|_{H^{1}\left(\mathcal{A}_{\rho}\right)}=0 .
$$

For any $u, v \in H^{1}\left(\mathcal{A}_{R}\right)$, it will be useful to define the following semidefinite positive hermitian product:

$$
\langle u, v\rangle_{R}=\operatorname{Re} \int_{\mathcal{A}_{R}} \nabla u \cdot \nabla \bar{v} d x
$$

and the associated seminorm

$$
[u]_{R}=\langle u, v\rangle_{R}^{\frac{1}{2}}
$$

We have the following Lemma.

Lemma 2.2. Let $u, v \in H^{1}\left(\mathcal{A}_{R}\right)$ and let

$$
u(r, \omega)=\sum_{n \in \mathbb{Z}} u_{n}(r) e^{i n \omega}, \quad v(r, \omega)=\sum_{n \in \mathbb{Z}} v_{n}(r) e^{i n \omega} .
$$


Then we have that

$$
\langle u, v\rangle_{R}=\sum_{n \in \mathbb{Z}} \int_{R_{0}}^{R}\left[\rho u_{n}^{\prime}(\rho) \bar{v}_{n}^{\prime}(\rho)+\frac{n^{2}}{\rho} u_{n}(\rho) \bar{v}_{n}(\rho)\right] d \rho .
$$

Proof. Let $u, v \in C^{1}\left(\mathcal{A}_{R}\right)$. From (2.2), we obtain that

$$
\nabla u(r, \omega)=\sum_{n \in \mathbb{Z}}\left[u_{n}^{\prime}(r) \mathbf{e}_{r}+i n \frac{u_{n}(r)}{r} \mathbf{e}_{\omega}\right] e^{i n \omega},
$$

and an analogous formula holds for $v$. Fubini-Tonelli's Theorem and Parseval's identity yield (2.12). If $u, v \in H^{1}\left(\mathcal{A}_{R}\right)$, then the conclusion follows from a standard approximation argument.

\section{Convergence estimates}

In this section we prove our main result on the convergence of the approximating solution. Our strategy is to write a minimization problem for solutions of the homogeneous Helmholtz equation which is equivalent to Problem (2.8) and then we use Fourier representation to obtain an explicit expression of the minimizer.

For any function $u \in H^{1}\left(\mathcal{A}_{R}\right)$, we define $U \in H^{1}\left(\mathcal{A}_{R}\right)$ as follows:

$$
U(x)=e^{-i k|x|} u(x) .
$$

By using this notation, the functional $J_{R}$ in (2.8) can be written as

$$
J_{R}(u)=\langle U, U\rangle_{R}=[U]_{R}^{2} .
$$

In the following lemma we write a minimization problem for solutions of the homogeneous Helmholtz equation which is equivalent to Problem (2.8).

Lemma 3.1. For a fixed $R>R_{0}$, let $u_{\mathcal{A}_{R}}$ be the minimizer of Problem (2.8) and set

$$
v_{\mathcal{A}_{R}}=u_{\mathcal{A}_{R}}-\psi,
$$

with $\psi$ given by (2.6). Then, $v_{\mathcal{A}_{R}}$ is the unique minimizer of the following problem

$$
\text { Minimize } I_{R}(v):=\langle V+2 \Psi, V\rangle_{R},
$$

where $v$ is a solution of

$$
\begin{cases}\Delta v+k^{2} v=0, & \text { in } \mathcal{A}_{R} \\ v=0, & \text { on } \partial B_{R_{0}}\end{cases}
$$

here $V$ and $\Psi$ are the functions associated to $v$ and $\psi$ by (3.1), respectively.

Proof. For any $u \in H^{1}\left(\mathcal{A}_{R}\right)$, we define $v \in H^{1}\left(\mathcal{A}_{R}\right)$ by $v=u-\psi$. Hence the functional $J_{R}$ in (2.8) is given by

$$
J_{R}(v+\psi)=\langle\Psi, \Psi\rangle_{R}+\langle V+2 \Psi, V\rangle_{R},
$$

where $v$ is a solution of (3.4). Since $\langle\Psi, \Psi\rangle_{R}$ is fixed, we conclude.

Thanks to Lemma 3.1, we can find an explicit formula for $v_{\mathcal{A}_{R}}$. In particular, we have the following theorem. 
Theorem 3.2. Let $v_{\mathcal{A}_{R}}$ be the minimizer of Problem (3.3). Then,

$$
v_{\mathcal{A}_{R}}(r, \omega)=\sum_{n \in \mathbb{N}} v_{n}^{R} \eta_{n}(k r) e^{i n \omega},
$$

where

$$
\eta_{n}(\rho)=Y_{n}\left(k R_{0}\right) J_{n}(\rho)-J_{n}\left(k R_{0}\right) Y_{n}(\rho), \quad \rho>0,
$$

and

$$
v_{n}^{R}=-\frac{f_{n} \gamma_{n}^{R}}{c_{n}^{R}},
$$

with

$$
c_{n}^{R}=\int_{R_{0}}^{R}\left[\rho k^{2} \eta_{n}^{\prime}(k \rho)^{2}+\left(\rho k^{2}+\frac{n^{2}}{\rho}\right) \eta_{n}(k \rho)^{2}\right] d \rho,
$$

and

$$
\begin{aligned}
& \gamma_{n}^{R}=\frac{2}{\pi} k i\left(R-R_{0}\right)+ \\
& +\frac{1}{H_{n}^{(1)}\left(k R_{0}\right)} \int_{R_{0}}^{R}\left[k^{2} \rho H_{n}^{(1) \prime}(k \rho) \eta_{n}^{\prime}(k \rho)+\left(k^{2} \rho+\frac{n^{2}}{\rho}\right) H_{n}^{(1)}(k \rho) \eta_{n}(k \rho)\right] d \rho .
\end{aligned}
$$

Proof. Since $u_{\mathcal{A}_{R}}$ solves (2.9), then $v_{\mathcal{A}_{R}}=u_{\mathcal{A}_{R}}-\psi$ solves (3.4). By separation of variables and from the homogeneous boundary condition on $\partial B_{R_{0}}$, we write a solution $v$ of (3.4) as

$$
v(r, \omega)=\sum_{n \in \mathbb{N}} v_{n} \eta_{n}(k r) e^{i n \omega},
$$

where $\eta_{n}$ is given by (3.6). Since $V(r, \omega)=e^{-i k r} v(r, \omega)$, then

$$
V(r, \omega)=\sum_{n \in \mathbb{N}} v_{n} \tilde{\eta}_{n}(k r) e^{i n \omega},
$$

where we set

$$
\tilde{\eta}_{n}(r)=e^{-i k r} \eta_{n}(k r) .
$$

By letting $\Psi(r, \omega)=e^{-i k r} \psi(r, \omega)$, we have that

$$
\Psi(r, \omega)=\sum_{n \in \mathbb{N}} \frac{f_{n} \tilde{h}_{n}(k r)}{H_{n}^{(1)}\left(k R_{0}\right)} e^{i n \omega},
$$

where

$$
\tilde{h}_{n}(r)=e^{-i k r} H_{n}^{(1)}(k r) .
$$

We notice that

$$
\tilde{\eta}_{n}^{\prime}(\rho)=k e^{-i k \rho}\left(\eta_{n}(k \rho)-i \eta_{n}(k \rho)\right)
$$

from Lemma 2.2 and since $\eta_{n}$ is real-valued, we have that

$$
\langle V, V\rangle_{R}=\sum_{n \in \mathbb{N}}\left|v_{n}\right|^{2} c_{n}^{R},
$$

where $c_{n}^{R}$ is given by (3.8). Analogously, from

$$
\langle\Psi, V\rangle_{R}=\operatorname{Re} \sum_{n \in \mathbb{N}} \frac{f_{n} \bar{v}_{n}}{H_{n}^{(1)}\left(k R_{0}\right)} \bar{v}_{n} \int_{R_{0}}^{R}\left[\rho \tilde{h}_{n}^{\prime}(\rho) \overline{\tilde{\eta}_{n}^{\prime}(\rho)}+\frac{n^{2}}{\rho} \tilde{h}_{n}(\rho) \overline{\tilde{\eta}_{n}(\rho)}\right] d \rho,
$$


we obtain that

$$
\begin{aligned}
& \text { 11) } \rho \tilde{h}_{n}^{\prime}(\rho) \overline{\tilde{\eta}_{n}^{\prime}(\rho)}+\frac{n^{2}}{\rho} \tilde{h}_{n}(\rho) \overline{\tilde{\eta}_{n}(\rho)}= \\
& =\rho k^{2}\left(H_{n}^{(1) \prime}(k \rho)-i H_{n}^{(1)}(k \rho)\right)\left(\eta_{n}^{\prime}(k \rho)+i k \eta_{n}(k \rho)\right)+\frac{n^{2}}{\rho} H_{n}^{(1)}(k \rho) \eta_{n}(k \rho) .
\end{aligned}
$$

Some computations yield $H_{n}^{(1) \prime}(k \rho) \eta_{n}(k \rho)-H_{n}^{(1)}(k \rho) \eta_{n}^{\prime}(k \rho)=H_{n}^{(1)}\left(k R_{0}\right)\left[J_{n}(k \rho) Y_{n}^{\prime}(k \rho)-J_{n}^{\prime}(k \rho) Y_{n}(k \rho)\right]$, and, from

$$
J_{n}(r) Y_{n}^{\prime}(r)-J_{n}^{\prime}(r) Y_{n}(r)=\frac{2}{\pi r}
$$

(see formula 9.1.16 in [AS]), we obtain that

$$
H_{n}^{(1) \prime}(k \rho) \eta_{n}(k \rho)-H_{n}^{(1)}(k \rho) \eta_{n}^{\prime}(k \rho)=\frac{2}{\pi k \rho} H_{n}^{(1)}\left(k R_{0}\right) .
$$

From (3.11) and (3.12) we have that

$$
\langle\Psi, V\rangle_{R}=\operatorname{Re} \sum_{n \in \mathbb{N}} f_{n} \gamma_{n}^{R} \bar{v}_{n}
$$

with $\gamma_{n}^{R}$ given by (3.9) and hence

$$
\langle V+2 \Psi, V\rangle_{R}=\sum_{n \in \mathbb{N}} c_{n}^{R}\left|v_{n}\right|^{2}+2 \operatorname{Re} f_{n} \gamma_{n}^{R} \bar{v}_{n} .
$$

By minimizing each term of the sum we obtain (3.7).

In order to obtain estimates on the convergence, it will be useful to write the coefficients $c_{n}^{R}$ and $\gamma_{n}^{R}$ more explicitly. We will need the following lemma.

Lemma 3.3. Let $\mathcal{C}_{n}$ and $\mathcal{D}_{n}$ be two cylinder functions, with $n \in \mathbb{N}$. Then

$$
\begin{aligned}
& \int^{r}\left[\rho \mathcal{C}_{n}^{\prime}(\rho) \mathcal{D}_{n}^{\prime}(\rho)+\left(\rho+\frac{n^{2}}{\rho}\right) \mathcal{C}_{n}(\rho) \mathcal{D}_{n}(\rho)\right] d \rho= \\
& r^{2}\left(\mathcal{C}_{n}(r) \mathcal{D}_{n}(r)+\mathcal{C}_{n}^{\prime}(r) \mathcal{D}_{n}^{\prime}(r)\right)+r \mathcal{C}_{n}^{\prime}(r) \mathcal{D}_{n}(r)-n^{2} \mathcal{C}_{n}(r) \mathcal{D}_{n}(r) .
\end{aligned}
$$

Proof. We multiply the Bessel equation

$$
\left(r \mathcal{C}_{n}^{\prime}(r)\right)^{\prime}+\left(r-\frac{n^{2}}{r}\right) \mathcal{C}_{n}(r)=0
$$

times $\mathcal{D}(r)$ and integrate. After one integration by parts, we have that

$$
r \mathcal{C}_{n}^{\prime}(r) \mathcal{D}_{n}(r)-\int^{r} \rho \mathcal{C}_{n}^{\prime}(\rho) \mathcal{D}_{n}^{\prime}(\rho) d \rho+\int^{r}\left(\rho-\frac{n^{2}}{\rho}\right) \mathcal{C}_{n}(\rho) \mathcal{D}_{n}(\rho) d \rho=0,
$$

and hence

$$
\int^{r} \rho \mathcal{C}_{n}^{\prime}(\rho) \mathcal{D}_{n}^{\prime}(\rho) d \rho+\int^{r}\left(\rho+\frac{n^{2}}{\rho}\right) \mathcal{C}_{n}(\rho) \mathcal{D}_{n}(\rho) d \rho=r \mathcal{C}_{n}^{\prime}(r) \mathcal{D}_{n}(r)+2 \int^{r} \rho \mathcal{C}_{n}(\rho) \mathcal{D}_{n}(\rho) d \rho
$$


From formula 10.22.5 in OLBC we obtain

$$
\begin{aligned}
& (3.14) \quad \int^{r} \rho \mathcal{C}_{n}^{\prime}(\rho) \mathcal{D}_{n}^{\prime}(\rho) d \rho+\int^{r}\left(\rho+\frac{n^{2}}{\rho}\right) \mathcal{C}_{n}(\rho) \mathcal{D}_{n}(\rho) d \rho= \\
& =r \mathcal{C}_{n}^{\prime}(r) \mathcal{D}_{n}(r)+\frac{r^{2}}{2}\left(2 \mathcal{C}_{n}(r) \mathcal{D}_{n}(r)-\mathcal{C}_{n-1}(r) \mathcal{D}_{n+1}(r)-\mathcal{C}_{n+1}(r) \mathcal{D}_{n-1}(r)\right) .
\end{aligned}
$$

By using the recurrence relations 10.6.2 in [OLBC], we find that

$$
\mathcal{C}_{n-1}(r) \mathcal{D}_{n+1}(r)+\mathcal{C}_{n+1}(r) \mathcal{D}_{n-1}(r)=2 \mathcal{C}_{n}^{\prime}(r) \mathcal{D}_{n}^{\prime}(r)+2 \frac{n^{2}}{r^{2}} \mathcal{C}_{n}(r) \mathcal{D}_{n}(r),
$$

and from (3.14) we conclude.

Now we are ready to find estimates of the rate of convergence of the solution of the approximating problem to the exact solution.

Theorem 3.4. Let $N \in \mathbb{N}$ and $R_{*}>R_{0}$ be fixed. Let $\psi$ and $u_{\mathcal{A}_{R}}$ be the solutions of (2.1) and (2.8), respectively, and assume that

$$
f(\omega)=\sum_{n=-N}^{N} f_{n} e^{i n \omega},
$$

with $\omega \in[0,2 \pi]$. Then

$$
\left\|\psi-u_{\mathcal{A}_{R}}\right\|_{H^{1}\left(\mathcal{A}_{R_{*}}\right)}=\mathcal{O}\left(R^{-1}\right),
$$

and

$$
\left\|\psi-u_{\mathcal{A}_{R}}\right\|_{H^{1}\left(\mathcal{A}_{R}\right)}=\mathcal{O}\left(R^{-1 / 2}\right),
$$

as $R \rightarrow+\infty$.

Proof. It will be enough to estimate the rate of convergence of the solution of $v_{\mathcal{A}_{R}}$ to zero, which clearly gives the desired $H^{1}$ estimate of the difference between the exact and the approximating solutions, as follows from (3.2).

Let $n \in \mathbb{N}$ be fixed and let $c_{n}^{R}$ and $\gamma_{n}^{R}$ be given by (3.8) and (3.9). We use Theorem 3.2, Lemma 3.3, and the asymptotic formulas in Section 10.17 in [OLBC] and we find the following asymptotic expansions for $R \rightarrow+\infty$ :

$$
c_{n}^{R}=\frac{2 k R}{\pi}\left[J_{n}\left(k R_{0}\right)^{2}+Y_{n}\left(k R_{0}\right)^{2}\right]+O(1)
$$

and

$$
\begin{aligned}
& \frac{\pi}{2} \gamma_{n}^{R}=-i k R_{0}-k^{2} R_{0}^{2} \frac{H_{n}^{(1)^{\prime}}\left(k R_{0}\right)}{H_{n}^{(1)}\left(k R_{0}\right)} \eta_{n}^{\prime}\left(k R_{0}\right)+ \\
& +\frac{e^{i \chi_{n}}}{H_{n}^{(1)}\left(k R_{0}\right)}\left[\alpha_{n} H_{n}^{(2)}\left(k R_{0}\right) e^{i \chi_{n}}+i\left(Y_{n}\left(k R_{0}\right) \cos \chi_{n}-J_{n}\left(k R_{0}\right) \sin \chi_{n}\right)\right]+ \\
& +O\left(R^{-1}\right),
\end{aligned}
$$

where

$$
\alpha_{n}=\frac{\left(4 n^{2}-1\right)\left(n^{2}-1\right)}{2},
$$

and

$$
\chi_{n}=k R-\left(n+\frac{1}{2}\right) \frac{\pi}{2}
$$


In particular we have that

$$
\frac{\gamma_{n}^{R}}{c_{n}^{R}}=O\left(R^{-1}\right), \quad \text { as } R \rightarrow+\infty
$$

Since $R_{*}$ is fixed, then there exists a constant $C$, not depending on $R$ such that

$$
\left\|\eta_{n}\right\|_{H^{1}\left(\mathcal{A}_{R_{*}}\right)} \leq C
$$

for every $n$, and hence it is clear that (3.17) implies (3.15). To prove (3.16) we notice that formula 10.22.5 in [OLBC] implies that

$$
\int_{0}^{R}\left(\eta_{n}(r)^{2}+\eta_{n}^{\prime}(r)^{2}\right) r d r=O(R), \quad \text { as } R \rightarrow+\infty,
$$

and from (3.17) we conclude.

Remark 3.5. In Theorem 3.4 we assumed that the source $f$ can be expressed in terms of a finite sum of Fourier coefficients, which is the most interesting case for the numerical computations. For a general $f \in L^{2}$ it is not clear whether Theorem 3.4 holds. Indeed, the error bounds that we used for the asymptotic expansions of cylindric functions may be not sufficient to guarantee the convergence (see Section 10.17(iii) in [OLBC]) and a more refined argument is probably needed to estimate the rate of convergence of the approximating solution.

\section{REFERENCES}

[AK] H. Ammari and H. Kang, Reconstruction of small inhomogeneities from boundary measurements. Lecture Notes in Mathematics, 1846. Springer-Verlag, Berlin, 2004.

[AS] M. Abramowitz and I. A. Stegun, Handbook of mathematical functions. Dover, New York, 1965.

[AO] O. Alexandrov and G. Ciraolo, Wave propagation in a 3-D optical waveguide. Math. Models Methods Appl. Sci. (M3AS), 14 (2004), no.6, 819-852.

[Be] P. Bettess, Infinite Elements, Penshaw Press, Sunderland, UK, 1992.

[Ber] J.-P. Bérenger. A perfectly matched layer for the absorption of electromagnetic waves, J. Comput. Phys. 114 (1994), 185-200.

[Ci1] G. Ciraolo, A method of variation of boundaries for waveguide grating couplers, Applicable Analysis, 87 (2008), 1019-1040.

[Ci2] G. Ciraolo, A radiation condition for the 2-D Helmholtz equation in stratified media, Comm. Part. Diff. Eq., 34 (2009), 1592-1606.

[CGS] G. Ciraolo, F. Gargano, V. Sciacca, A computational method for the Helmholtz equation in unbounded domains based on the minimization of an integral functional. Journal of Computational Physics, 246 (2013), 78-95.

[CGS2] G. Ciraolo, F. Gargano, V. Sciacca, A spectral approach to a constrained optimization problem for the Helmholtz equation in unbounded domains, to appear in Computational \& Applied Mathematics.

[CM1] G. Ciraolo and R. Magnanini. Analytical results for 2-D non-rectilinear waveguides based on the Green's function. Math. Methods Appl. Sci., 31 (2008), no.13, 1587-1606.

[CM2] G. Ciraolo and R. Magnanini, A radiation condition for uniqueness in a wave propagation problem for 2-D open waveguides, Math. Methods Appl. Sci., 32 (2009), $1183-1206$.

[CK] D. Colton and R. Kress Inverse Acoustic and Electromagnetic Scattering Theory, Springer-Verlag Berlin, Heidelberg, New York, 1992.

[EM] B. Engquist and A. Majda. Radiation boundary conditions for acoustic and elestic wave calculations, Comm. Pure Appl. Math., 32 (1979), 314-358.

[Gi] D. Givoli, Numerical Methods for Problems in Infinite Domains, Studies in Applied Mechanics, vol. 33, Elsevier Scientific. Publishing Co., Amsterdam, 1992. 
[GrK] M.J. Grote, J.B. Keller, On nonreflecting boundary conditions, J. Comput. Phys., 122 (1995), 231-243.

[Har] I. Harari, A survey of finite element methods for time-harmonic acoustics, Comput. Methods Appl. Mech. Engrg., 195 (2006), 1594-1607.

[Har] F. Ihlenburg, textitFinite Element Analysis of Acoustic Scattering, Springer-Verlag, New York, 1998.

[KG] J.B. Keller and D. Givoli. Exact nonreflecting boundary conditions, J. Comput. Phys., 82 (1989), 172-192.

[MS] R. Magnanini and F. Santosa, Wave propagation in a 2-D optical waveguide, SIAM J. Appl. Math., 61 (2001), 1237-1252.

[MTH] M. Medvinsky, E. Turkel and U. Hetmaniuk, Local absorbing boundary conditions for elliptical shaped boundaries, J. Comput. Phys. 227 (2008), no. 18, 8254-8267.

[OLBC] F. W. Olver, D. W. Lozier, R. F. Boisvert and C. W. Clark, NIST Handbook of Mathematical Functions, Cambridge University Press, New York, NY, USA, 2010.

$[\mathrm{PV}]$ B. Perthame and L. Vega. Energy concentration and Sommerfeld condition for Helmholtz equation with variable index at infinity, GAFA Geom. Funct. Anal., 17 (2008), 1685-1707.

[SS] S. A. Sauter and C. Schwab, Boundary element methods, Springer Series in Computational Mathematics, 39, Springer-Verlag, Berlin, 2011.

Dipartimento di Matematica e Informatica, Università di Palermo, Via ArchiRAFi 34, 90123 PAlermo, ItAly

E-mail address: giulio.ciraolo@unipa.it 